\title{
Design of Weak 1-D Bragg Grating Filters in SOI Waveguides Using Volume Holography Techniques
}

\author{
Marie Verbist, Wim Bogaerts, Member, IEEE, and Dries Van Thourhout, Member, IEEE
}

\begin{abstract}
To answer the growing need for more versatile integrated spectral filters, we show that weak one-dimensional gratings can be designed towards any desired target spectrum. We follow a very straightforward design procedure to demonstrate the performance of these devices. Measurements and simulations show a very good correspondence with the target spectra. By analyzing the results, we also found that the design procedure can be refined by using simulated reflections, instead of relying on the calculated Fresnel reflections.
\end{abstract}

\section{INTRODUCTION}

$\mathbf{F}$ ABRICATION technologies for photonic integrated circuits, such as optical lithography and ebeam lithography, are becoming more and more refined They are constantly enabling more functionality and complexity on photonic chips. One required functionality for many applications is a more $\mathrm{fl} \mathrm{x}$ ible and versatile spectral filte. Current devices based on photonic crystals, arrayed waveguide gratings or ring resonators are well understood and have excellent performance [1], [2], but these approaches lack versatility.

The principles of volume holography [3], [4] present an answer to the growing need for dedicated spectral filter with complex transfer functions. By allowing light to travel over a large distance through a weak grating, the light spectrum can be manipulated in a very specifi manner [5].

As silicon-on-insulator (SOI) offers many advantages for photonic integrated circuits, we aim to integrate this type of grating in a silicon waveguide. As this material system has a very high refractive index contrast, the difficult is to obtain sufficien control over the fabrication technology to cause a very small effective refractive index variation with the required accuracy. As gratings offer many possibilities [6], [7], we propose to implement these weak gratings by modulating the shallow etched sides of a ridge waveguide [8].

In our previous work [9], we presented the weak grating theory on which these gratings are based, and demonstrated the performance of the firs prototypes. In this paper, we discuss the fabrication limits, based on a larger set of devices and we compare the measurement results with simulation results.

Manuscript received June 13, 2013; revised December 4, 2013 and January 20, 2014; accepted April 1, 2014. Date of publication April 9, 2014; date of current version April 27, 2014.

The authors are with the Department of Information Technology, Ghent University and Interuniversity Microelectronics Center, 9000 Ghent, Belgium (e-mail: marie.verbist@intec.ugent.be; wim.bogaerts@intec.ugent.be; dries.vanthourhout@intec.ugent.be).

Color versions of one or more of the f gures in this paper are available online at http://ieeexplore.ieee.org.

Digital Object Identifie 10.1109/JLT.2014.2316478
Furthermore, the design procedure is analyzed, leading to improvements for future devices.

\section{WEAK GRATING THEORY}

We represent a grating structure by the fiel reflectio $R(x)$ along the propagation direction $x$. When a pulse $E_{0} \delta(t)$ is incident on this grating, it produces a reflectio $E_{R}(t)$ at the input port, which depends on $R(x)$. To obtain the spectral transfer function of this grating, the Fourier transform of $E_{R}(t) / E_{0}$ has to be calculated:

$$
T(f)=\mathcal{F}\left[\frac{E_{R}(t)}{E_{0}}\right] .
$$

For the design of holographic gratings, two important assumptions are made. Firstly, we assume the gratings are weak enough to ignore multiple reflections as is often assumed when considering weak fibe gratings [10]. The pulse $E_{0} \delta(t)$ then interacts with this weak grating by producing a single reflectio at every point $x$, with an amplitude of $E_{0} R(x)$. When this reflec tion reaches the input port, it will be delayed by a transit time $t$ of

$$
t=\frac{2 n_{0} x}{c}
$$

where $n_{0}$ is the average background index of the grating [4]. For simplicity, we have not taken into account material and waveguide dispersion, which means $n_{0}$ is a constant value, equal to the effective refractive index, in the following calculation. The spectral transfer function of this weak grating becomes

$$
T(f)=\mathcal{F}\left[R\left(\frac{c t}{2 n_{0}}\right)\right] .
$$

The second assumption is that the reflectio $R(x)$ of this reflectio solely depends on the effective refractive index variation $\Delta n$ at that point, and can be calculated as a Fresnel reflection

$$
R(x)=\frac{\Delta n(x)}{2 n_{0}} .
$$

The validity of this assumption will be discussed in the further analysis (part VII). The spectral transfer function then becomes

$$
T(f)=\mathcal{F}\left[\frac{1}{2 n_{0}} \Delta n\left(\frac{c t}{2 n_{0}}\right)\right] .
$$

Vice versa, the effective index variation $\Delta n(x)$ that will result in the reflectio of a specifi target spectrum $T(f)$, can be calculated as the inverse Fourier transform of $T(f)$. The effective index $n(x)=n_{0}+\Delta n(x)$ that is needed to obtain this reflectio spectrum, can thus be calculated as

$$
n(x)=n_{0}+c_{0} \mathcal{F}^{-1}[T(f)] .
$$



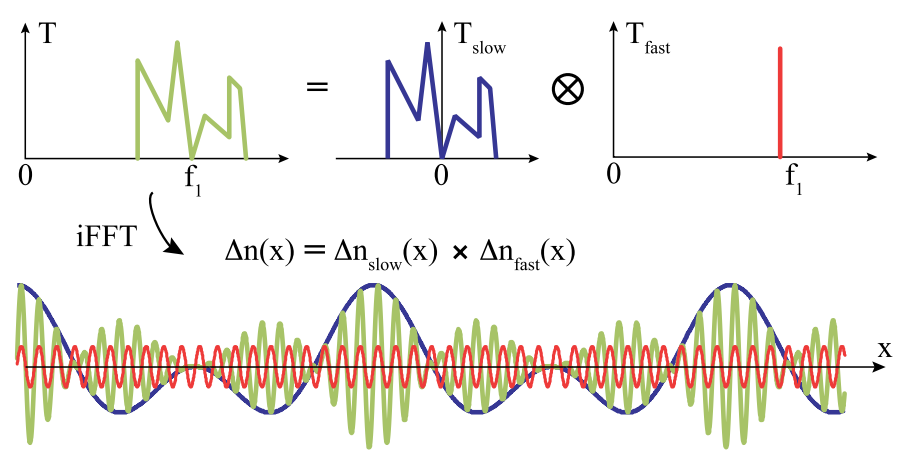

Fig. 1. The target spectrum is translated into the corresponding $\Delta n(x)$ by means of an inverse Fourier transform. Spectra situated around the same frequency range, exhibit the same fast period. The slow modulation depends on the shape of the spectrum. The $\Delta n(x)$ shown here is in case the spectrum consists of a number of isolated peaks, resulting in a superposition of sines.

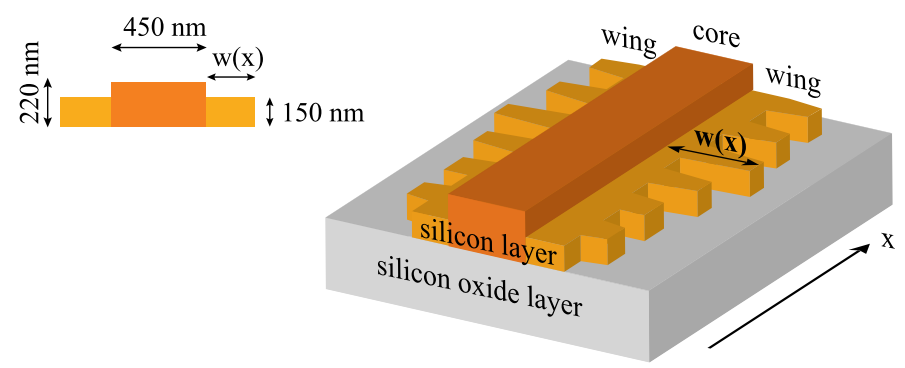

Fig. 2. The used SOI ridge waveguides consist of a silicon core ( $220 \mathrm{~nm}$ high) and shallow etched silicon wings $(150 \mathrm{~nm}$ high). The holographic gratings are implemented by modulating the width $w$ of these wings along the propagation direction $x$.

We introduce the constant $c_{0}$ to control the maximal $\Delta n$. Note that $n_{0}$ can be chosen freely within the limits of the SOI material system, taking only practical considerations into account.

To test this concept, several target spectra were translated into the corresponding $\Delta n(x)$. The spectra consist of a set of isolated peaks, leading to a superposition of sines for $\Delta n(x)$. Fig. 1 shows how a random target spectrum consist of a fast period and a slow modulation. In the case of isolated peaks around $1550 \mathrm{~nm}, \Delta n(x)$ exhibits a fast period of about $290 \mathrm{~nm}$, which depends on the choice of $n_{0}$, and a slow modulation, which depends on the relative positions of the peaks.

\section{IMPLEMENTATION}

In principle, the implementation of the effective refractive index variation is arbitrary, and can be chosen to fi the aspired application and available fabrication techniques. In this paper, we start from a double-etched ridge waveguide, and implement the holographic grating as a modulation of the width of the shallow etched sides, referred to as wing width $w(x)$ [8]. This is illustrated in Fig. 2. The central part of the waveguide measures $220 \mathrm{~nm}$ high by $450 \mathrm{~nm}$ wide. The silicon layer in the wing is $150 \mathrm{~nm}$ high.

The relation between the effective refractive index $n$ and the wing width $w$ was simulated with the full-vectorial mode solver Fimmwave. We obtain the function $w(n)$, as shown in Fig. 3(b). At this point, a choice has to be made regarding the strength

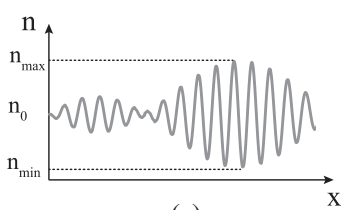

(a)

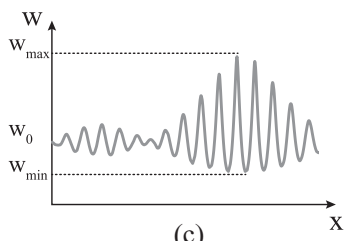

(c)

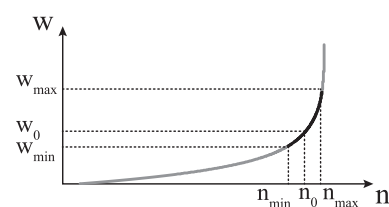

(b)

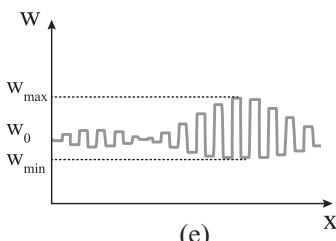

(e)
Fig. 3. (a) The effective refractive index $n$ as a function of the propagation direction $x$ is calculated as the inverse fourier transform of the target spectrum. (b) The relation between the wing width $w$ and $n$ is simulated in the mode solver Fimmwave. (c) From $n(x)$ and $w(n)$ follows the original design $w(x)$. (d) The original design is transformed into a mask layout that can be fabricated using deep-UV lithography.
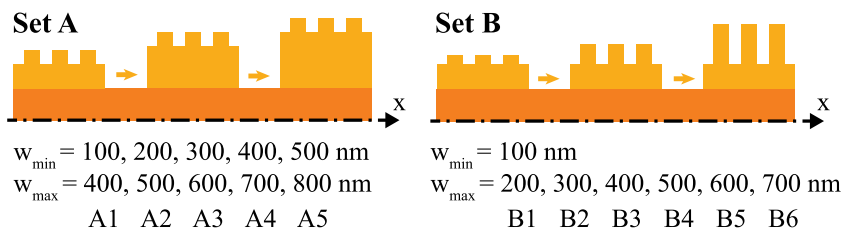

Fig. 4. These top views represent the two sets of $w_{\min }$ and $w_{\max }$ that were used to implement the holographic gratings. This allows us to investigate fabrication issues and the spectral behavior related to grating strength.

of the grating, define by the parameter $c_{0}$, and the background refractive index $n_{0}$. This is equivalent to fixin $w_{\min }$ and $w_{\max }$.

To investigate the fabrication and the behavior of the holographic gratings, two sets of $w_{\min }$ and $w_{\max }$ were used. They are visualized by the top views in Fig. 4. For set A we have chosen $w_{\text {min }}$ from $100 \mathrm{~nm}$ to $500 \mathrm{~nm}$, while keeping the maximal $\Delta w=w_{\max }-w_{\min }$ constant at $300 \mathrm{~nm}$. The corresponding maximal $\Delta n$ ranges from 0.098 to 0.003 . For a set $\mathrm{B}, w_{\min }$ is kept at $100 \mathrm{~nm}$, while $w_{\max }$ ranges from 100 to $600 \mathrm{~nm}$. The corresponding maximal $\Delta n$ ranges from 0.063 to 0.105 .

\section{FABRICATION}

\section{A. From Original Design to Mask Layout}

Depending on the fabrication scheme, additional transformations can or should be performed to satisfy fabrication requirements. For this study, the photonic chips are fabricated using $193 \mathrm{~nm}$ deep-UV lithography [11]. The original design is therefore translated to a mask layout. Features narrower than $120 \mathrm{~nm}$ are not allowed by the software design rule check as they cannot be fabricated accurately. Therefore, we apply a transformation that truncates all sharp features by replacing $\mathrm{w}(\mathrm{x})$ with the average value over this $120 \mathrm{~nm}$ range [see Fig. 3(d)]. As the fast grating period is about $290 \mathrm{~nm}$, the shape of the gratings in the mask layout appears rectangular, even though in between the truncated parts, the sine function is maintained.

For example, the designs in set $\mathrm{A}$ all had an original $\Delta w$ of $300 \mathrm{~nm}$. On the mask layout, the truncation reduces the value of $\Delta w$ to about $150 \mathrm{~nm}$. Obviously, this transformation reduces 

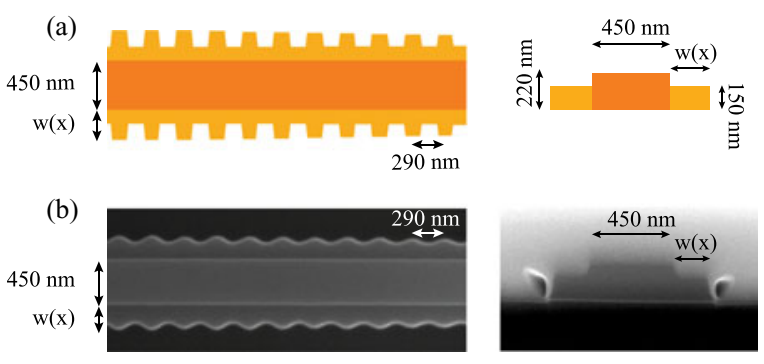

Fig. 5. The mask layout (a) is compared to a SEM image of the fabricated device (b). Both a top view (left) and a cross-section (right) are shown.
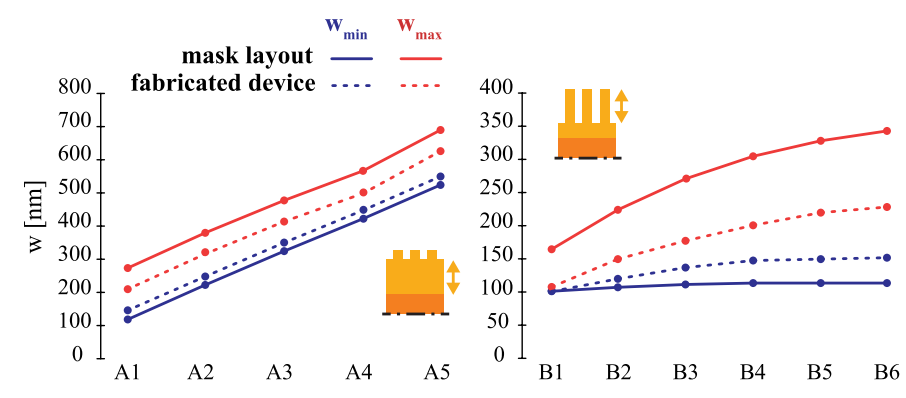

Fig. 6. For both sets of devices, the measured values of $w_{\min }$ and $w_{\max }$ (dotted line) show a significan difference with the values in the mask layout (full line). Note that the mask layout is in fact the truncated original design.

the maximal $\Delta n$ of the gratings. On average, the maximal $\Delta n$ is reduced to about $63 \%$ of its original value.

\section{B. From Mask Layout to Fabricated Device}

Fig. 5 shows a comparison of one of the mask layouts and the corresponding fabricated device. As we expect from [12], the edges are rounded and the maximal wing width $w_{\max }$ has decreased. Measurements on the SEM image also reveal that the minimal wing width $w_{\min }$ has slightly increased. This all considerably reduces the effective strength of the grating.

We performed SEM measurements of $w_{\min }$ and $w_{\max }$ on all devices of set A and B. Fig. 6 compares these widths (dotted line) with the values in the mask layout (full line). Note that the mask layout is in fact the truncated version of the original design. The corresponding values for $\Delta n$ are reduced to about $38 \%$ of the values corresponding to the mask layout. As a consequence, throughout the entire process from original design to fabricated device, the value of $\Delta n$ is reduced to $24 \%$ of the value in the original design. Fig. 7 shows the values of $\Delta n$ for the original design, the mask layout and the fabricated device.

The fabricated devices of set $\mathrm{A}$ all have a constant $\Delta w$ of about $50 \mathrm{~nm}$, which indicates that the fabrication is not influ enced by the choice of $w_{\min }$. Set $\mathrm{B}$ shows that $w_{\min }$ is only accurately fabricated for the smallest $\Delta w$. For larger $\Delta w$, the value of $w_{\min }$ firs increases, and then remains constant. Fig. 8 shows the relation between the designed $\Delta w$ and the measured values after fabrication. For small $\Delta w$, the relation between design and fabrication is parabolic. From values of about $250 \mathrm{~nm}$, the relation becomes linear. This graph includes measurements of test devices with even higher values of $\Delta w$ than in set $\mathrm{A}$ and $\mathrm{B}$.
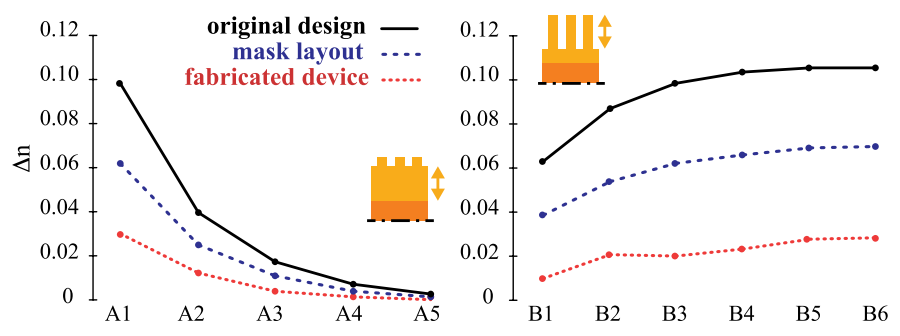

Fig. 7. By truncating the narrow features in the original design to obtain the mask layout, the effective refractive index contrast is reduced to $63 \%$ of the value in the original design. By fabricating the device, there is a reduction to $38 \%$ of the value in the mask design. As a consequence, in total, the effective refractive index of the fabricated device is about $24 \%$ of the value in the original design.

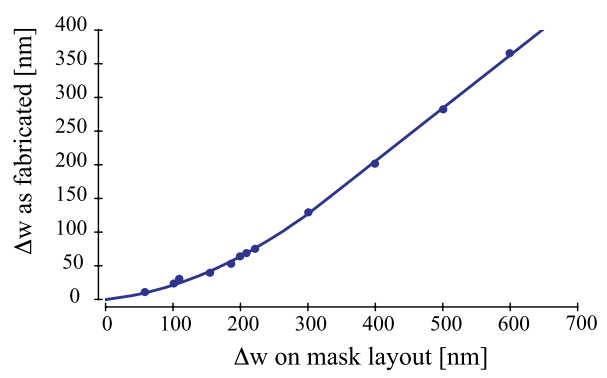

Fig. 8. The $\Delta w$ as measured in the fabricated devices is shown as a function of the $\Delta w$ from the mask layout. The relation is parabolic for values below $250 \mathrm{~nm}$, after which it becomes linear.

Future devices could benefi from reverse engineering: by using this data, the grating teeth in the design can be extended to compensate for the fabrication effects, resulting in the desired value of $\Delta w$. It should be noted that this relation will change if the period of the grating changes significantl .

\section{Measurements}

The waveguides are equipped with grating couplers to couple light from an optical fibe to the waveguide and back [13]. A tunable laser is used as a light source and the reflecte and transmitted power are measured simultaneously using two power meters. The reflecte power is measured through a circulator and index matching flui is used between fibe and chip to reduce unwanted reflections Polarization paddles control the polarization of the light. Both transmission and reflectio spectra are normalized with respect to the transmission of a straight waveguide with the same grating couplers. For the reflectio spectra, we also take the additional loss through the circulator into account.

Fig. 9 shows the measured reflectio and transmission spectra of four gratings with different target spectra and/or parameters:

1) one single peak,

2) three peaks, separated by $10 \mathrm{~nm}$,

3) three peaks, separated by $5 \mathrm{~nm}$, and one by $10 \mathrm{~nm}$,

4) nine peaks, separated by $5 \mathrm{~nm}$.

The measured spectra distinctly show the peaks that were set in the target spectra. There is a slight shift to smaller wavelengths and the peaks also appear slightly closer together than in the original design. The shift is a consequence of using a single value for $n_{0}$ in the design of the gratings, while in fact, $n_{0}$ 

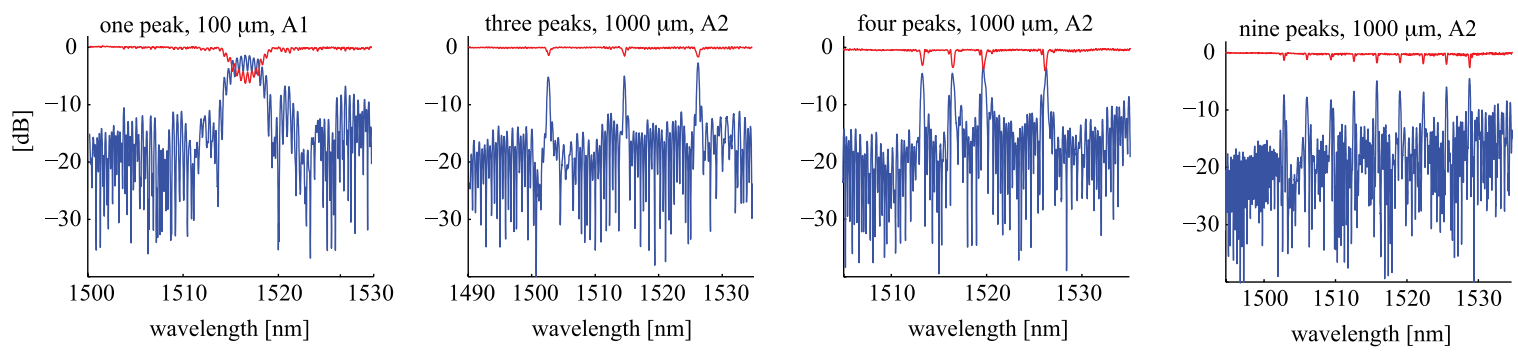

Fig. 9. Four examples of gratings with different spectra and parameters demonstrate the performance of the holographic gratings.
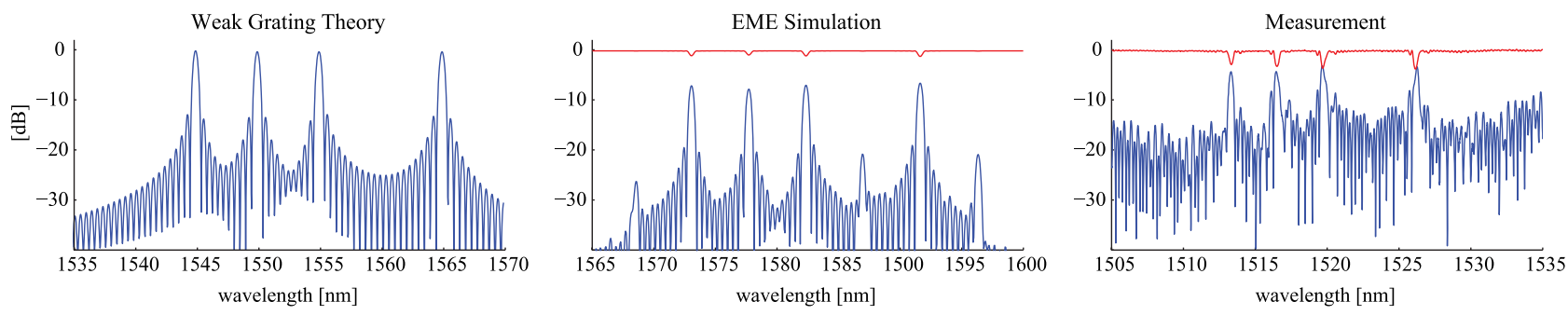

Fig. 10. The measured spectra of the four-peak spectrum with a length of $1000 \mu \mathrm{m}$ and parameters A2 show a very good correspondence with simulations, using the effective index method in Camfr, a mode solver based on EME, as well as calculations based on the weak grating theory (normalized).

is different for each specifi implementation. The spectrum is slightly compressed because $n_{0}$ does depend on the wavelength, which was not taken into account. This dependence could be incorporated into the design procedure, by using simulated values of $n_{0}(\lambda)$ in Eq. (2) of the design procedure, instead of the effective refractive index. This would increase the correspondence between the target spectrum and the measurements. However, by taking into account material and waveguide dispersion, the design and the implementation can no longer be independent from each other.

We analyze the measurements in more detail in Section VII.

\section{SimULATIONS}

First of all, the expected reflectio spectrum can be calculated by performing a forward Fourier transform on the fina $\Delta n$, taking into account the actual length of the grating and the truncation transformation. This provides a fast way of verifying for example that truncating the grating does not significantl changes the reflectio spectrum at the wavelengths of interest.

Obviously, this calculation based on the weak grating theory does not take the actual implementation as a ridge waveguide into account. Therefore, we need to import the actual devices into a physical simulation tool. We use the effective index method in Camfr, a mode solver base on eigenmode expansion (EME) [14]. As there is a considerable difference between the mask layout and the fabricated device, the measured wing widths were used.

Fig. 10 shows the calculated, simulated and measured spectra of the grating designed for the spectrum with three peaks, separated by $5 \mathrm{~nm}$, and one additional peak separated by $10 \mathrm{~nm}$. The grating has a length of $1000 \mu \mathrm{m}$ and the parameters from A2. The effective index method slightly overestimates the effective index of the waveguide. For example, for a wing width of $100 \mathrm{~nm}$, the effective index method returns a refractive in- dex of 2.65, while the two-dimensional mode solver Fimmwave calculates a value of 2.53. This causes the simulated spectra to shift to larger wavelengths. The index constrast is also slightly lower, which results in smaller reflection and thus lower dips. Apart from this, the simulated spectra show a very good correspondence with the measurements. We also see a good correspondence between the simulation in Camfr, and the theoretical calculation based on the weak grating theory. This indicates, that - at least in this particular case - the assumptions of the weak grating theory hold. The interstitial peaks that are visible in the simulation, should also be present in the measurements, but we suspect they don't rise above the noise level.

\section{FURTHER ANALYSIS}

The resolution of a grating is determined by both its length and strength. The weakest and longest grating have the highest spectral resolution. In our case of weak reflectio gratings, there is a clear tradeoff between length, resolution and reflecte power.

Limiting the length of a grating can be interpreted as multiplying the infinit grating with a rectangular window. In the Fourier domain, this causes a convolution of the peak with a sinc function. The shorter the rectangular window, the broader this sinc function becomes. This translates into a lower spectral resolution. Fig. 11 shows the 3-peak spectrum for increasing grating lengths. The peaks become narrower until a grating length of about $500 \mu \mathrm{m}$, after which they remain constant. This width $\Delta \lambda$ is limited by the strength of the grating.

To investigate the spectral resolution in more detail, we take a closer look at the gratings with a target spectrum consisting of one single peak. For such a regular Bragg grating, following relation between the fiel reflectio $R$ at a single interface and the spectral resolution holds:

$$
\Delta \lambda=\frac{R}{2 \pi} \lambda
$$



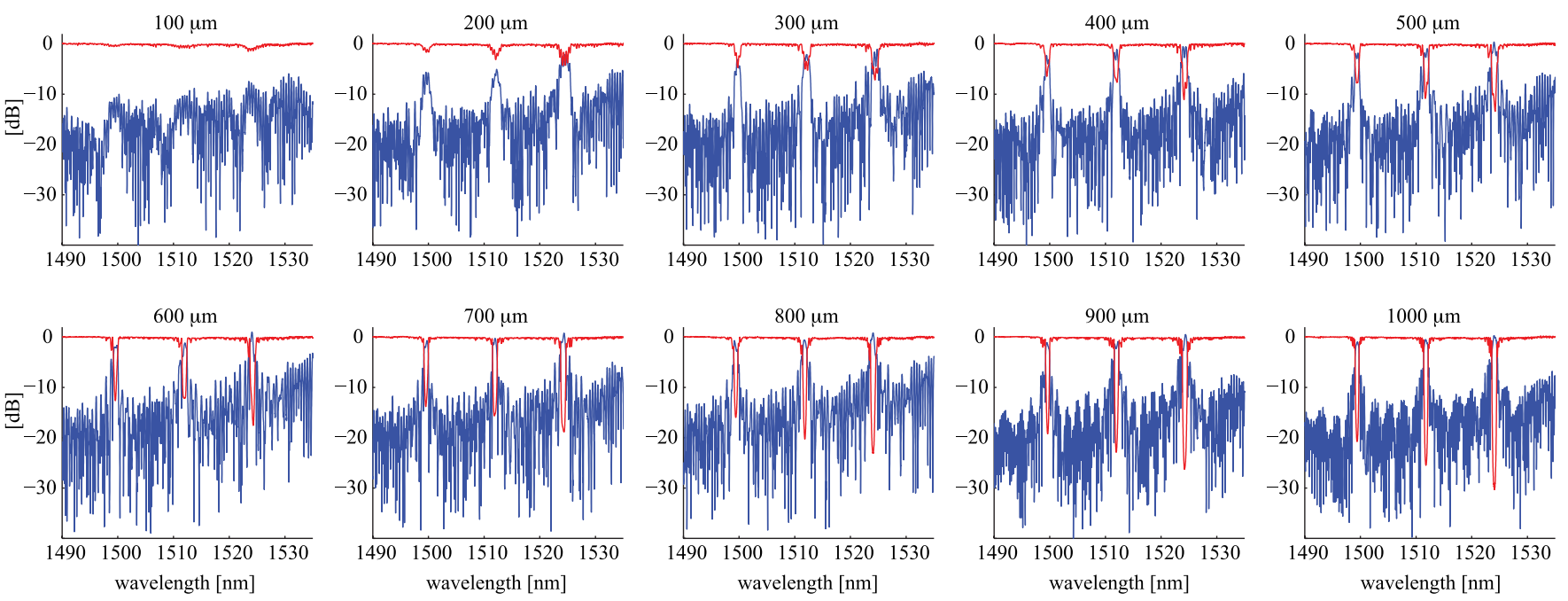

Fig. 11. The peak widths of this three-peak spectrum (with parameters A1) decrease for increasing grating length until a length of about $500 \mu \mathrm{m}$, after which they remain constant.

A1

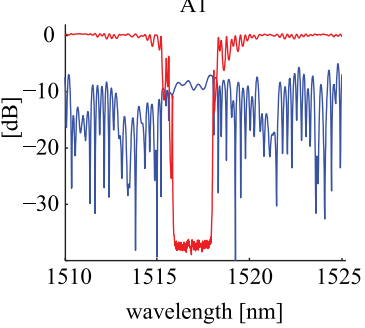

A2

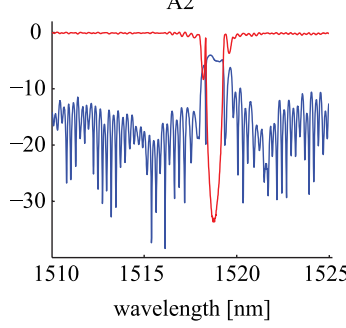

A3

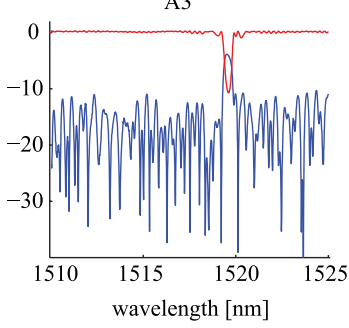

A4

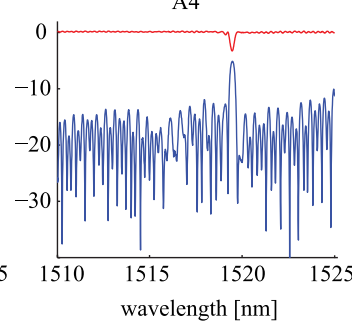

A5

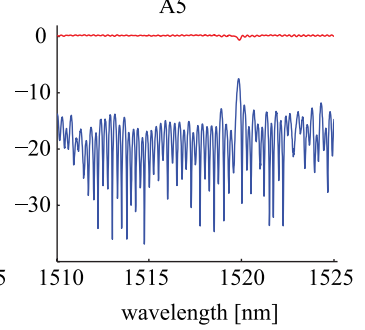

Fig. 12. Reducing the grating strength clearly increases the resolution.
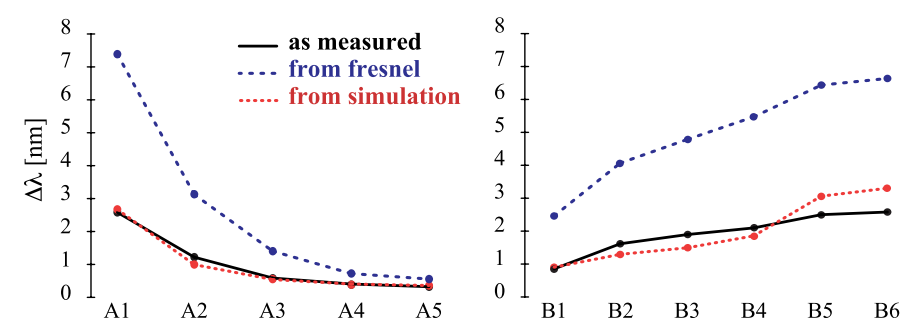

Fig. 13. The expected peak widths $\delta \lambda$ calculated based on the Fresnel reflec tion is about a factor 3 larger than the measured peak widths. The peak widths calculated based on the simulated reflectio do match the measurements for the devices of both set $\mathrm{A}$ and set $\mathrm{B}$.

Fig. 13 shows $\Delta \lambda$ as measured, as calculated based on the Fresnel reflectio [Eq. (4)], and as calculated based on the simulated reflectio in Camfr. It is clear the measurement curve corresponds very well with the simulated curve, but $\Delta \lambda$ based on the Fresnel reflectio is approximately a factor 3 larger. To illustrate this discrepancy even further, Fig. 14 shows the reflectio at a single interface between a waveguide with wing width $w_{\text {min }}$ and $w_{\max }=w_{\min }+\Delta w$ calculated with the Fresnel equation, together with the same reflectio simulated in Camfr. The white lines indicate the physical dimensions of set $\mathrm{A}$ and set $\mathrm{B}$. Along these white lines, the difference in magnitude is about a factor 3 between both simulations. This can largely be attributed to the fact that in SOI photonic waveguides, the group refractive index is much higher than the effective refractive index [8]. Therefore,

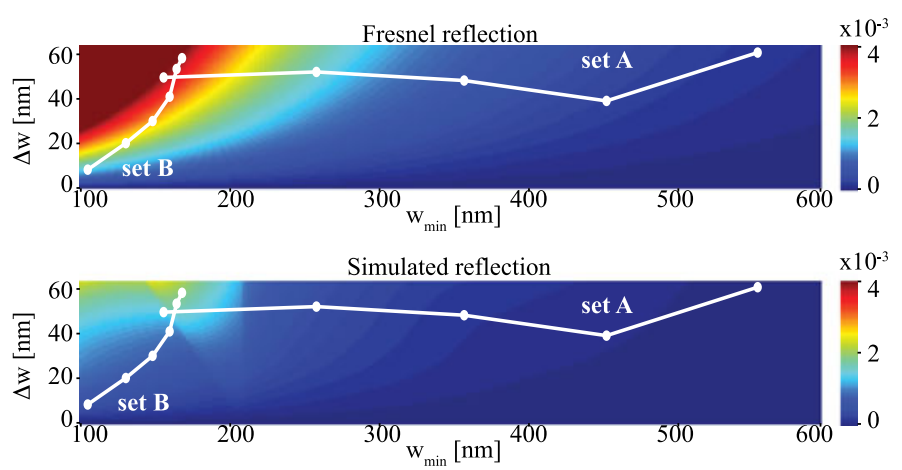

Fig. 14. The reflectio at a single interface between a waveguide with wing width $w_{\min }$ and a waveguide with wing width $w_{\max }=w_{\min }+\Delta w$ was calculated using the Fresnel equation, and simulated using the mode solver Camfr. The white lines connect the dots that represent the parameters from set $\mathrm{A}$ and set $\mathrm{B}$.

in Eq. (4), $n_{0}$ should be replaced with the group index $n_{g}$. Furthermore, as the index change is not stepwise but sinusoidal, the grating strength is reduced by a factor of $p i / 4$.

Fig. 14(b) also shows a noticeably different pattern than Fig. 14(a). We believe this is caused by the changing shape of the modes, and the changing number of supported modes in the waveguide. These changes influenc the amount of light that is reflected 
Given the above considerations, we can improve our design cycle by removing the approximation $R(x)=\frac{\Delta n(x)}{2 n_{0}}$ (Eq. (4)) from the theory. Instead of implementing the required $\Delta n(x)$, the required $R(x)$ should be implemented. To do this, instead of the simulated function $n(w)$, the simulated $R\left(w_{0}, \Delta w\right)$ should be used directly to translate between theory and implementation. Without substantially complicating the design cycle, this adjustment should ensure more accurate results.

\section{CONCLUSION}

We have shown that a weak grating can be designed to produce a variety of tailored reflectio spectra. The design procedure takes this target spectrum as its input, and returns the required weak effective refractive index variation $\Delta n$, which can then be implemented in any suitable way. In our case, we chose to modulate the shallow etched side wings of a ridge waveguide. Gratings were fabricated using deep UV lithography, for several target spectra and several grating strengths and lengths. Both measurements and simulations confir that this is a promising approach to integrate $\mathrm{fl}$ xible and versatile filter on photonic chips to enable more complex applications.

When investigating the resolution of the gratings in more detail, we found that it would be more accurate to directly implement the required fiel reflectio $R(x)$. Furthermore, taking waveguide dispersion into account and improving the truncation algorithm will improve correspondence with the target spectrum.

\section{ACKNOWLEDGMENT}

The authors acknowledge ePIXfab for the fabrication of the SOI chips. M. Verbist acknowledges the Institute for the Promotion of Innovation through Science and Technology in Flanders (IWT Vlaanderen) and the Methusalem project "Smart Photonic Chips" of Ghent University.

\section{REFERENCES}

[1] T. Tsuchizawa, K. Yamada, H. Fukuda, T. Watanabe, J. Takahashi, M. Takahashi, T. Shoji, E. Tamechika, S. Itabashi, and H. Morita, "Microphotonics devices based on silicon microfabrication technology," IEEE J. Sel. Topics Quantum Electron., vol. 11, no. 1, pp. 232-240, Jan./Feb. 2005.

[2] W. Bogaerts, S. K. Selvaraja, P. Dumon, J. Brouckaert, K. De Vos, D. Van Thourhout, and R. Baets, "Silicon-on-insulator spectral filter fabricated with CMOS technology," IEEE J. Sel. Topics Quantum Electron., vol. 16, no. 1, pp. 33-44, Jan./Feb. 2010.

[3] C. Peroz, A. Goltsov, S. Dhuey, P. Sasorov, B. Harteneck, I. Ivonin, S. Kopyatev, S. Cabrini, S. Babin, and V. Yankov, "High-resolution spectrometer-on-chip based on digital planar holography," IEEE Photon. J., vol. 3, no. 5, pp. 888-896, Oct. 2011.

[4] T. Mossberg, "Planar holographic optical processing devices," Opt. Lett., vol. 26, no. 7, pp. 414-416, 2001.

[5] Y. Huang, V. Baby, P. Prucal, C. Greiner, D. Iazikov, and T. Mossberg, "Integrated holographic encoder for wavelength-hopping/time-spreading optical CDMA," IEEE Photon. Technol. Lett., vol. 17, no. 4, pp. 825-827, Apr. 2005

[6] W. Shi, H. Yun, C. Lin, J. Flueckiger, N. Jaeger, and L. Chrostowski, "Coupler-apodized bragg-grating add-drop filte," Opt. Lett., vol. 38, pp. 3068-3070, 2013.

[7] A. D. Simard, N. Belhadj, Y. Painchaud, and S. LaRochelle, "Apodized silicon-on-insulator bragg gratings," IEEE Photon. Technol. Lett., vol. 24, no. 12 , pp. 1033-1035, Jun. 15, 2012.
[8] X. Wang, W. Shi, H. Yun, S. Grist, N. Jaeger, and L. Chrostowski, "Narrowband waveguide bragg gratings on SOI wafers with CMOS-compatible fabrication process," Opt. Exp., vol. 20, pp. 15547-15558, 2012.

[9] M. Verbist, D. Van Thourhout, and W. Bogaerts, "Weak gratings in siliconon-insulator for spectral filter based on volume holography," Opt. Lett., vol. 38, no. 3, pp. 386-388, 2013.

[10] J. Sancho, S. Chin, D. Barrera, S. Sales, and L. Thevenaz, "Time-frequency analysis of long fibe bragg gratings with low reflect vity," Opt. Exp., vol. 21, no. 6, pp. 7171-7179, 2013.

[11] S. K. Selvaraja, P. Jaenen, W. Bogaerts, D. Van Thourhout, P. Dumon, and R. Baets, "Fabrication of photonic wire and crystal circuits in silicon-oninsulator using 193-nm optical lithography," J. Lightw. Technol., vol. 27 , no. 18, pp. 4076-4083, Sep. 2009.

[12] X. Wang, W. Shi, M. Hochberg, K. Adam, E. Schelew, J. Young, N. Jaeger, and L. Chrostowski, "Lithography simulation for the fabrication of silicon photonic devices with deep-ultraviolet lithography," in Proc. IEEE 9th Int. Conf. Group IV Photon, 2012, pp. 288-90.

[13] D. Vermeulen, S. Selvaraja, P. Verheyen, G. Lepage, W. Bogaerts, P. Absil, D. Van Thourhout, and G. Roelkens, "High-efficien y fibe -to-chip grating couplers realized using an advanced CMOS-compatible silicon-oninsulator platform," Opt. Exp., vol. 18, no. 17, pp. 18278-18283, 2010.

[14] P. Bienstman and R. Baets, "Optical modelling of photonic crystals and VCSELs using eigenmode expansion and perfectly matched layers," Opt. Quantum Electron., vol. 33, pp. 327-341, 2001.

Marie Verbist received the degree in electrotechnical engineering from the Ghent University, Ghent, Belgium, in 2008. She is currently working toward the $\mathrm{Ph} . \mathrm{D}$. degree in engineering at the Photonic Research Group, Ghent UniversityIMEC, Ghent, Belgium. Her current research interests include the fabrication of nanophotonic structures based on focused ion-beam etching and the modeling, design and fabrication of holographic gratings.

Dries Van Thourhout received the degree in physical engineering and the Ph.D. degree in applied physics from Ghent University, Ghent, Belgium, in 1995 and 2000 , respectively.

From October 2000 to September 2002, he was with Lucent Technologies, Bell Laboratories, Murray Hill, NJ, USA, where he was involved in the designing, processing, and characterization of InP/InGaAsP monolithically integrated devices. In October 2002, he joined the Department of Information Technology, Ghent University, where he is currently a Member of the permanent staff of the Photonics Group. He is a Lecturer or Colecturer within the Ghent University Master in Photonics program and coordinates the cleanroom activities of the research group. He is also engaged in the development of new fabrication processes for photonic devices. He holds four patents. He has authored and coauthored more than 120 journal papers and invited papers at several major conferences. His research interests include the design, fabrication, and characterization of integrated photonic devices, silicon nanophotonic devices, and heterogeneous integration of InP-on-silicon.

Dr. Van Thourhout is a Member of IEEE Laser and Electrooptics Society and an Associate Editor for IEEE Photonics Technology Letters.

Wim Bogaerts completed his studies in engineering (applied physics) at Ghent University, Belgium, in 1998, and joined the Department of Information Technology (INTEC) at both Ghent University and the Interuniversity Microelectronics Center (IMEC), where he received the Ph.D. degree in 2004. In the Photonics Research Group, he specialized in the modeling, design, and fabrication of nanophotonic components. He is currently a Professor in the fiel of silicon photonics at Ghent University, and coordinates the activities between IMEC and Ghent University in silicon photonics design.

Dr. Bogaerts is a Member of the IEEE Photonics Society, the Optical Society of America, and SPIE. 